\title{
Performance Analysis on Half Iris Feature Extraction using GW, LBP and HOG
}

\author{
G. Savithiri \\ Research Scholar, \\ Mother Terasa Women's University, \\ Kodaikanal.
}

\author{
A.Murugan \\ Dept. of Computer Science, \\ Dr. Ambedkar Govt. Arts College, \\ Chennai - 39, India.
}

\begin{abstract}
Iris recognition is the most accurate biometrics which has received increasing attention in departments which require high security. In this paper, we discussed Gabor Wavelet, Local Binary Pattern, Histogram of Oriented Gradient techniques to extract features on specific portion of the iris for improving the performance of an iris recognition system. The main aim of this paper is to show that is enough to choose the half portion of the iris to recognize authentic users and to reject imposters instead of whole extension of the iris. The proposed methods are evaluated based upon False Rejection Rate (FRR) and False Acceptance Rate (FAR) and the experimental results show that this technique produces good performance on MMU iris database.
\end{abstract}

\section{Keywords}

Biometrics, Iris Recognition, Gabor Wavelet, Local Binary Pattern, Histogram of Orientation Gradient

\section{INTRODUCTION}

Biometrics is the science of establishing human identity by using physical or behavioral traits such as face, fingerprints, palm prints, iris, hand geometry and voice. Iris recognition systems, in particular, are gaining interest because the iris's rich texture offers a strong biometric cue for recognizing individuals. No two iris patterns are alike, even those of identical twins, even between the right and left eye of the same person [3]. From one year of the age until death, the patterns of the iris are relatively constant over a person's lifetime [3][10].

Information from iris patterns is dispersed randomly and non-uniformly over the region of the iris, but on the notices that there is a concentration of characteristics at the region near the pupil. Due to this great quantity of information good recognition efficiency can be achieved with only 50\% or less of the iris region available for analysis [2]. The goal of this paper is to show that it is possible to improve the reliability of the system by choosing only half portion of the iris region. We discussed three feature extraction techniques such as Gabor Wavelet, Local Binary Pattern and Histogram of Oriented Gradient on iris recognition by two different methods, first by taking internal region, which is near to the pupil and external region. The internal region gives high recognition rate than external region [22]. The second methods divide the iris as upper half and lower half portion to identify individuals. Here, lower portion gives good recognition rate than upper portion of the iris.
The features extracted from the human iris can be used to identify person [15], even among genetically identical twins. Gabor Wavelet transform is used for analyzing the human iris patterns and extracting feature points from them. Phase data from 1D Gabor is extracted and quantized to four levels to encode the unique patterns of iris into a bitwise biometric template. In this work, the local binary patterns (LBP) are adopted to represent texture patterns of iris images. The LBP has emerged as an effective texture descriptor and is widely used in texture analysis. The original LBP, introduced by Ojala [8] is defined for each pixel by comparing its $3 \times 3$ neighborhood pixels with the centre pixel value and considering the result as a binary bit string. Due to its discriminative power and computational simplicity, LBP texture operator has become a popular approach in various applications. It can be seen as a unifying approach to the traditionally divergent statistical and structural models of texture analysis. Iris has very fine textures and local rotations, affine transformations and distortions of the iris image are common phenomenon. Normalized iris image is divided into sub-regions according to the properties of iris textures. Local binary patterns (LBP) are then adopted for texture representation of each sub-region. Next, the Histogram of Oriented Gradient (HOG) feature vectors for the detecting widow are computed as feature based on appearance. Recently, several appearance feature [4]-[6] for detecting people were proposed. In particular Dalal etal [5] presented a people detection algorithm that has an excellent detection ability. Each detection window is divided into cells of size $8 \times 8$ pixels and each group of $2 \times 2$ cells is integrated into a block in a sliding fashion, so the blocks overlap. Each cells consisting of a 9-bin Histogram of Oriented Gradients (HOG), each block contains a concatenated vector of all its cells. HOG features are invariant to illumination and local geometrical changes, many recent studies have used them to detect people. This paper is organized as follows. In Section II, the iris processing stages that include preprocessing, iris localization from the original eyes, normalization and enhancement are described; Section III discusses various feature extraction techniques. Section IV deals with iris matching based on Hamming Distance and Euclidean Distance. Experimental Results are reported in Section V. Conclusion is drawn in Section VI. 


\section{PROCESSING STAGES}

The overall system structure of the iris recognition includes image acquisition and iris recognition. The iris image acquisition includes the lighting system; the positioning system and the physical capture system. The iris recognition includes preprocessing, iris localization, normalization and iris template matching.

\subsection{Image Preprocessing}

Standard noise reduction and isolated peak noise removal techniques such as median-filtering and average filtering used to clear the noise and to make the iris textures good and clear. In practical applications of a workable system an image of the eye to be analyzed must be acquired first in digital form suitable for analysis. We transformed the images from RGB to gray level for further processing and used MMU iris image database available in the public domain.

\subsection{Localization of the Iris Region}

Integro-differential operator is used for locating the inner and outer boundaries of iris as well as the upper and lower eyelids [1][3]. The operator computes the partial derivative of the average intensity of circle points with respect to increasing radius $r$. After convolving the operator with Gaussian kernel, the maximum difference between the inner and outer circle will define the center and radius of the iris boundary. For upper and lower eyelids detection, the path of contour integration is modified from circular to parabolic curve. The operator is accurate because it searches over the image domain for the global maximum. It can compute faster because it uses the first derivative information.

\subsection{Iris Normalization}

Irises from different people may be captured in different size and even for the iris from the same person; the size may change because of the variation of the illumination and other factors such elastic deformations in iris texture affects the results of iris matching. For the purpose of achieving more accurate recognition results, the homogeneous rubber sheet model devised by Daugman [1] used to remap each point within the iris region to a pair of polar co-ordinates $(r$, $\theta$ ) where $r$ is on the interval $[0,1]$, upper half portion of the iris takes the angle $[0, \pi]$ and lower half portion takes the angle $[\pi, 2 \pi]$. The remapping of the iris region $\mathrm{I}(\mathrm{x}, \mathrm{y})$ from raw co-ordinates $(\mathrm{x}, \mathrm{y})$ to the doubly dimensionless nonconcentric polar co-ordinate system ( $r, \theta)$ can be represented as

$$
\begin{array}{r}
(\mathrm{I}(\mathrm{x}(\mathrm{r}, \theta), \mathrm{y}(\mathrm{r}, \theta)) \rightarrow \mathrm{I}(\mathrm{r}, \theta) \\
\text { with } \mathrm{x}(\mathrm{r}, \theta)=(1-\mathrm{r}) \mathrm{x}_{\mathrm{p}}(\theta)+\mathrm{rx}_{1}(\theta) \\
\mathrm{y}(\mathrm{r}, \theta)=(1-\mathrm{r}) \mathrm{y}_{\mathrm{p}}(\theta)+\mathrm{ry}_{1}(\theta)
\end{array}
$$

where $\mathrm{I}(\mathrm{x}, \mathrm{y})$ is the iris region image $(\mathrm{x}, \mathrm{y})$ are the original Cartesian co-ordinates, (r, $\theta)$ are the corresponding normalized polar co-ordinates and $\mathrm{x}_{\mathrm{p}}, \mathrm{y}_{\mathrm{p}}$ and $\mathrm{x}_{1}, \mathrm{y}_{1}$ are the co-ordinates of the pupil and iris boundaries along the $\theta$ direction. The rubber sheet model which takes into account pupil dilation and size inconsistencies in order to produce a normalized representation with constant dimensions.

\subsection{Iris Enhancement}

The normalized iris image has low contrast and nonuniform illumination caused by the light source position. The image needs to be enhanced to compensate for these factors. Local histogram analysis is applied to the normalized iris image to reduce the effect of non-uniform illumination and obtain well-distributed texture image [7][8]. Reflections regions are characterized by high intensity values close to 255. A simple thresholding operation can be used to remove the reflection noise.

\section{FEATURE EXTRACTION}

The iris is a thin circular part which lies between the pupil and sclera upper part of the pupil is usually occluded by top eyelid and eyelashes which their intensity is similar to pupil. On the contrary the lower part of pupil is rarely affected by any eyelids and eyelashes. The features of the iris can be extracted by different approaches for iris recognition is shown in the Figure 1. In the first method, iris region is divided into two ring regions as inner region

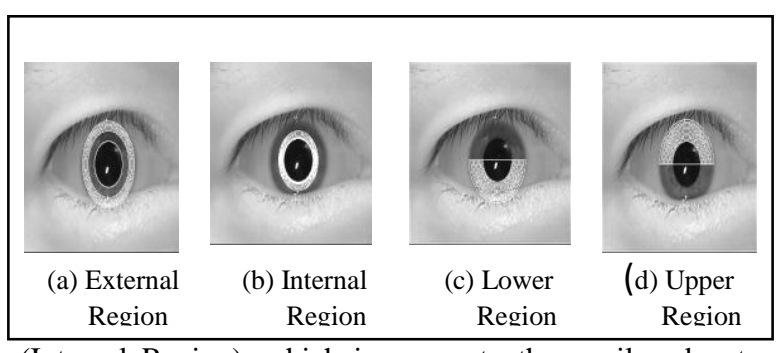

(Internal Region), which is nearer to the pupil and outer region (External Region) [22].

\section{Fig 1: Different approaches of Iris Recognition}

The region near the pupil (Inner Region) means that only points from this region is used to generate the rectangular representation. In this work, the angular and radial resolutions are set as 240 and 10 pixels respectively. Two bits are used to represent the quantized phase information for each pixel. Therefore, total size of the iris template is 4800 bits. In the second method, irises are taken as upper half and lower half portion for iris recognition. Here, the angular and radial resolutions are set as 120 and 20 pixels respectively. Hence the total size of the iris template is 4800 bits. The system's performance is evaluated considering each of those regions separately with different feature extraction techniques.

\subsection{Gabor Wavelet Features}

Gabor transform used for analyzing the human iris patterns and extracting feature points from them. In order to extract the discriminating features from the normalized collarets region, the normalized pattern is convolved with 1D Gabor wavelet. Thus feature encoding is implemented by first breaking the two-dimensional normalized iris pattern into one-dimensional wavelets and then these signals are convolved with 1D Gabor wavelet. The resulting phase information for both real and imaginary response is quantized, generating a bit wise template. The input eye 
image is in gray scale of Bit Depth $=8$ and Size $=320 \times 240$ and polar co-ordinates image size is $240 \times 20$ that will be the input dimension for feature extraction, then the output dimension would be 480x20.

\subsection{Local Binary Pattern (LBP) based Iris Recognition}

Local Binary Pattern features have performed very well in various applications including texture classification and segmentation, image retrieval and surface inspections. The LBP texture analysis operator is defined as a gray-scale invariant texture measure derived from a general definition of texture in a local neighborhood.

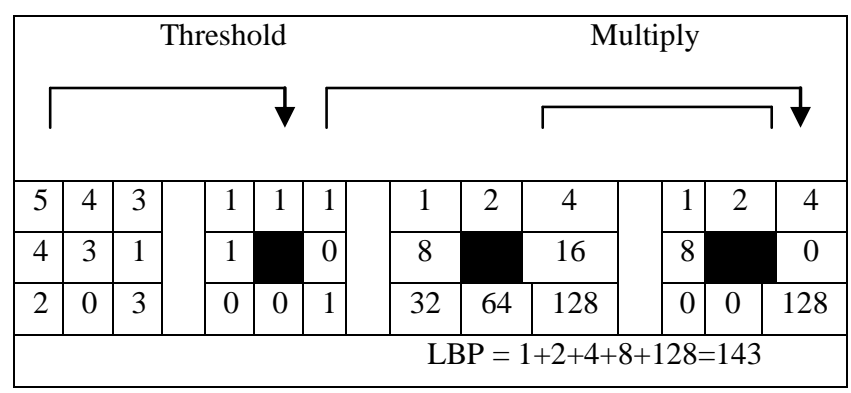

Fig 2: Calculating the original LBP code and a contrast measure

A LBP code for an eight neighborhood of a pixel is produced by multiplying the threshold values with weights given to the corresponding pixels and summing up the result. Since the LBP, by definition was invariant to monotonic changes in gray scale, it was supplemented by an orthogonal measure of local contrast. Figure 2 shows how the contrast measure was derived. The average of the gray levels below the center pixel is subtracted from that of the gray levels above (or equal to) the center pixel. The 256 bin histogram of the labels computed over an image can be used as a texture descriptor. Two dimensional distributions of the LBP and local contrast measures are used as features. Each iris images can be considered as a composition of micro-patterns which can be effectively detected by the LBP operator. The iris is divided into $M$ small nonoverlapping regions $R_{0}, R_{1}, \ldots . . R_{M}$.

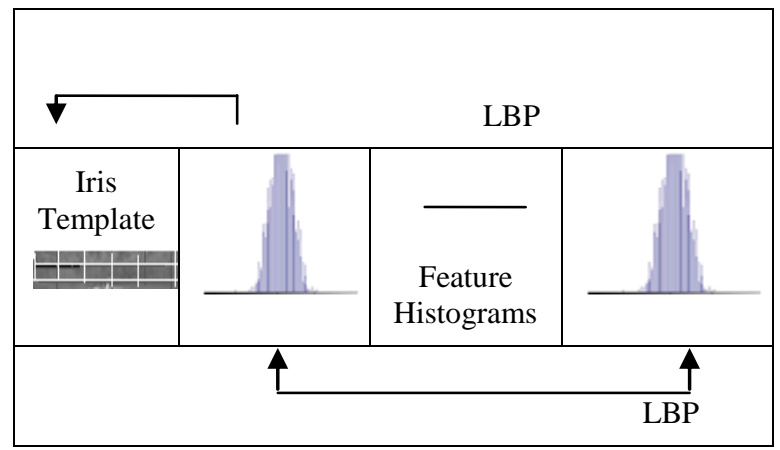

Fig 3: LBP based Iris Recognition

The LBP histograms extracted from each iris sub-regions as shown in the Figure 3, are then concatenated into a single, spatially enhanced feature histogram defined as

$$
\mathrm{H}_{\mathrm{i}, \mathrm{j}}=\sum_{\mathrm{x}, \mathrm{y}} \mathrm{I}\left(\mathrm{f}_{1}(\mathrm{x}, \mathrm{y})=\mathrm{i}\right) \mathrm{I}\left((\mathrm{x}, \mathrm{y}) \varepsilon \mathrm{R}_{\mathrm{j}}\right)
$$

Where $\mathrm{i}=0,1 \ldots \mathrm{L}-1, \mathrm{j}=0,1, \ldots \mathrm{M}-1$. The extracted feature histogram describes the local binary pattern for global iris images.

\subsection{Histogram of Oriented Gradients}

HOG are used as appearance features. HOG representation [5] has several advantages. It captures the gradient structure that is characteristic of the human shape. First magnitude M in equation (3) and orientation $\theta$ in equation (4) of the gradients are computed using

$$
\begin{gathered}
\left.\mathrm{M}(\mathrm{x}, \mathrm{y})=\operatorname{Sqrt}\left(\mathrm{f}_{\mathrm{x}}(\mathrm{x}, \mathrm{y})^{2}+\mathrm{fy}(\mathrm{x}, \mathrm{y})^{2}\right)\right) \\
\theta(\mathrm{x}, \mathrm{y})=\tan ^{-1}\left(\mathrm{f}_{\mathrm{y}}(\mathrm{x}, \mathrm{y}) / \mathrm{f}_{\mathrm{x}}(\mathrm{x}, \mathrm{y})\right)
\end{gathered}
$$

where $f_{x}(x, y)=L(x+1, y)-L(x-1, y), f_{y}(x, y)=L(x, y+1)-L(x, y-$ 1) and $\mathrm{L}(\mathrm{x}, \mathrm{y})$ is the brightness of pixel. Each detection window is divided into cells of size $5 \times 5$ pixels and each group of $2 \times 2$ cells is integrated into a block in a sliding fashion so that the blocks overlap with each other. Each cell consists of a 9-bin histogram of HOG features represented by $F_{i j}=\left[f_{1}, f 2 \ldots f_{9}\right]$. Each block contains a concatenated vector of all its cells. Each block is then represented by

$$
V_{k}=\left[F_{i j}, F_{i+1 j}, F_{i+2 j}, F_{i j+1}, F_{i+1 j+1}, F_{i+2 j+1}, F_{i j+2}, F_{i+1 j+2}, F_{i+2 j+2}\right] .
$$

The HOG feature of each iris scanning window is constituted by 141 gradient histograms extracted from $3 \times 47=141$ blocks (image patches of $10 \times 10$ pixels). This 5076 dimensional feature for full iris template is constituted by the sub-HOG of 141 blocks. The sub-HOG at each block are 36 dimensional vectors.

\section{TEMPLATE MATCHING}

Once the features are extracted using any one of the following techniques Gabor, LBP and HOG, an iris image is transformed into a unique representation within the feature space. In order to make the decision of acceptance or refusal, distance is calculated to measure the closeness to match the extracted features of the iris are compared with the iris images in the database. Here, two types of measures such as Hamming Distance and Euclidean Distance are used for classification.

\subsection{Hamming Distance}

This test enables the comparison of two iris patterns. This test is based on the idea that the greater the Hamming Distance between two Iris Codes A and B is defined as

$$
H D=\frac{\|(\operatorname{code} \mathrm{A} \otimes \operatorname{code} \mathrm{B}) \cap \operatorname{mask} \mathrm{A} \cap \operatorname{mask} \mathrm{B}\| .}{\|\operatorname{mask} \mathrm{A} \cap \operatorname{mask} \mathrm{B}\|} .
$$

The $\otimes$ operator is the Boolean XOR operation to detect disagreement between the pairs of phase code bits in the two Iris Codes (code A and code B), and mask A and B identify the values in each Iris Code that are not corrupted by artifacts such as eyelids/eyelashes and specularities. The $\cap$ operator is the Boolean AND operator. The $\|$. $\|$ operator is used to sum the number of "1" bits within its argument. This method corrects for misalignments in the normalized iris pattern caused by rotational differences 
during imaging. From the calculated distance values, the lowest one is taken. This serves as a measure of recognition performance, as it is the fractional Hamming Distance [2] that determines if identification has been made. To derive the performance results, each original iris image was compared against every other image in the database using the template matching technique.

\subsection{Euclidean Distance}

The Euclidean Distance is one way of defining the closeness of match between two iris feature templates. It is calculated between two templates vectors by

$$
\mathrm{P}_{1}\left(\mathrm{x}_{1}, \mathrm{y}_{1}\right) \text { and } \mathrm{P}_{2}\left(\mathrm{x}_{2}, \mathrm{y}_{2}\right)=\operatorname{Sqrt}\left\{\left(\mathrm{x}_{2}-\mathrm{x}_{1}\right)^{2}+\left(\mathrm{y}_{2}-\mathrm{y}_{1}\right)^{2}\right\}
$$

where $\mathrm{P}_{1}\left(\mathrm{x}_{1}, \mathrm{y}_{1}\right)$ is the first iris image with $\mathrm{x}$ and $\mathrm{y}$ coordinates of pixel, $\mathrm{P}_{2}\left(\mathrm{x}_{2}, \mathrm{y}_{2}\right)$ is the second iris image with $\mathrm{x}$ and $y$ coordinates of the pixel in equation (5). To get the distance value is by calculating the deviation between their co-ordinates. After that we square the root of the result. It is noted that we calculate the pixel value that located in the same co-ordinates.

To derive the performance results, each original image was compared against every other image in the database using the template matching technique.

\section{EXPERIMENTAL RESULTS}

In this section, the number of experiments was performed in order to evaluate the performance of the methods using Matlab 7.0 on an Intel Pentium IV $3.0 \mathrm{GHz}$ processor with 512 MB memory.

\subsection{MMU Iris Database}

Experiments are conducted on MMU iris database [10] and 130 images are chosen for the experiments. Those 130 images are divided into 26 classes and each of them has 5 images. The first image from each class is selected to be the template. The remaining 4 images of each class are adopted as the test set. To test the half iris recognition strategy using these images we have 260 possible cases to check the false rejection and 8125 possible cases for false acceptance. Different performance factors and thresholds are carefully chosen after intensive experimental evaluations to get the best performance.

\subsection{Recognition Analysis}

Two irises are determined whether from the same class by comparing the similarity between their corresponding feature vectors.

\subsubsection{Gabor Wavelet}

In IRIS Region first, phase data from 1D Gabor Wavelet was extracted and quantized to four levels to encode the unique pattern of the iris into a bit-wise biometric template as the feature vector. If the size of the feature vector is $\mathrm{L}$, then we represent an iris image with only $2 \mathrm{~L}$ bits. The iris feature vector length is of dimension $240 \times 20$ phase information for each pixel. Therefore, the total size of the iris template is 9600 bits. Second, the similarity between the two irises is calculated by using the exclusive OR operation. Using the Hamming Distance of two iris bit patterns, a decision can be made as to whether the two patterns were generated from different irises or from the same one. Hamming Distance [20][21] of $\leq 0.32$ allows identification with high confidence and used as threshold for recognition. If Hamming Distance $=0$ decides that it is perfect match between two iris codes. The experimental results indicate that the Hamming Distance [21] is between 0.16 and 0.32 for the iris images from the same eye and between 0.33 and 0.55 for the iris images from different eyes. In Internal and External Region, features extracted from the half iris template are of length 4800 bits i.e. $240 \times 10$ phase information for each pixel. In this work, Hamming Distance is between 0.16 and 0.38 for the iris images from the same eye and between 0.39 and above for the iris images from different eyes. The Hamming Distance is less than or equal to 0.1 for the iris images from the same eye and more than that indicates iris images from different eyes in Upper and Lower Region. Here, 120x20 phase information for each pixel is extracted as feature vectors. Hence, the total size of the template is 4800 bits.

\subsubsection{Local Binary Pattern}

Iris template is divided in various non-overlapping block regions of same size. The histogram of LBP codes is computed for each block. Each block histogram is thus normalized. The feature vectors of Gabor Wavelet, Local Binary Pattern and Histogram Oriented of Gradients are shown in the Table 1.

Table 1. Feature Vectors on Gabor, LBP, and HOG in dimension

\begin{tabular}{|c|c|c|c|}
\hline Method & $\begin{array}{c}\text { IRIS } \\
\text { Region }\end{array}$ & $\begin{array}{c}\text { External/Inter } \\
\text { nal Region }\end{array}$ & $\begin{array}{c}\text { Upper/Lower } \\
\text { Region }\end{array}$ \\
\hline Gabor & $240 X 20$ & $240 X 10$ & $120 \mathrm{X} 20$ \\
\hline LBP & $238 X 18$ & $238 X 8$ & $118 X 18$ \\
\hline HOG & $3 \times 47$ & 1 X47 & 3 X23 \\
\hline
\end{tabular}

\subsubsection{Histogram of Oriented Gradients \\ (HOG)}

HOG feature vectors for the detecting window are computed as feature based on appearance. Each detection window on iris template is divided into cells of size $10 \times 10$ pixels and each group of $5 \times 5$ cells is integrated into a block. In External and Internal region, the window is constituted by 47 gradient histograms extracted from $1 \times 47=47$ blocks. This 1692 dimensional feature is constituted by 141 blocks of sub-HOG. Each block is 36 dimensional vectors. The HOG feature of each scanning window in upper and lower portion of the iris region is 2484 dimensional feature and is constituted by the subHOG of 81 blocks.

\subsubsection{Result}

The proposed algorithms are tested in two modes: verification and identification. Correct Recognition Rate (CRR) is used to measure the algorithms in identification mode. In this paper, Gabor Wavelet (GW), Local Binary Pattern (LBP) and Histogram Oriented of Gradient (HOG) 
methods are compared with the existing algorithms proposed by Daugman [4], Li M.et.al [15], Tan et.al [16], etc are shown in the Table 2.

In verification mode, the Receiver Operating Characteristic (ROC) curve is plotted as the False Rejection Rate (FRR) against False Acceptance Rate (FAR) for an iris recognition system using GW, LBP and HOG are shown in the Figure 4, Figure 5 and Figure 6 respectively. For verification, a threshold is used to decide if the user is accepted or rejected.

Table 2. Performance Comparison of the GW, LBP, HOG with existing algorithms

\begin{tabular}{|c|c|c|c|}
\hline Algorithm & $\begin{array}{c}\text { FAR } \\
(\%)\end{array}$ & FRR (\%) & $\mathrm{CRR}(\%)$ \\
\hline Daugman [4] & 0.00 & 0.13 & 99.94 \\
\hline Li M.et. al [15] & 0.00 & 0.87 & 99.57 \\
\hline Tan et. al [16] & 0.00 & 1.03 & 99.49 \\
\hline Maheswari [17] & 0.01 & 0.88 & - \\
\hline L.V. Birgale [18] & 0.0071 & 1.0427 & 98.95 \\
\hline L V. Birgale [18] & 0.0069 & 1.0207 & 98.98 \\
\hline \multicolumn{4}{|c|}{ Proposed System Using Half Iris } \\
\hline $\begin{array}{l}\text { (A) Gabor Wavelet } \\
\text { (i) Iris Template }\end{array}$ & 0.006 & 0.00 & 99.94 \\
\hline ii) Inner Region & 0.008 & 1.15 & 99.84 \\
\hline (iii) Outer Region & 0.014 & 1.92 & 99.80 \\
\hline (iv) Upper Portion & 0.008 & 1.92 & 99.85 \\
\hline (v) Lower Portion & 0.018 & 2.69 & 99.73 \\
\hline $\begin{array}{c}\text { (B) Local Binary } \\
\text { Pattern } \\
\text { (i) Iris Template }\end{array}$ & 0.002 & 2.3 & 99.90 \\
\hline (ii) Inner Region & 0.004 & 3.85 & 99.83 \\
\hline (iii) Outer Region & 0.006 & 3.89 & 99.77 \\
\hline (iv) Upper Portion & 0.004 & 4.02 & 99.82 \\
\hline (v) Lower Portion & 0.008 & 7.03 & 99.68 \\
\hline $\begin{array}{l}\text { (C) Histogram } \\
\text { Oriented of } \\
\text { Gradient } \\
\text { (i) Iris Template }\end{array}$ & 0.00 & 1.50 & 99.95 \\
\hline (ii) Inner Region & 0.00 & 1.90 & 99.94 \\
\hline (iii) Outer Region & 0.08 & 2.69 & 99.83 \\
\hline (iv) Upper Portion & 0.00 & 2.3 & 99.92 \\
\hline (v) Lower Portion & 0.007 & 3.46 & 99.82 \\
\hline
\end{tabular}

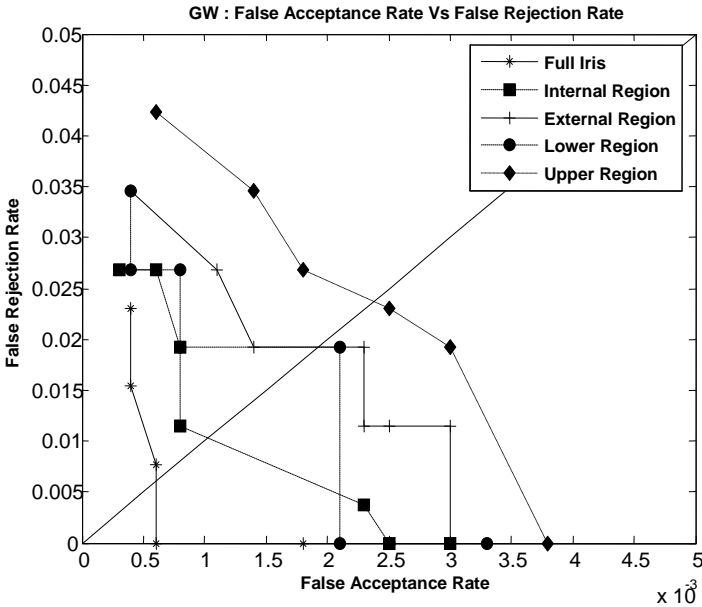

Fig 4: Gabor Wavelet: ROC Curve

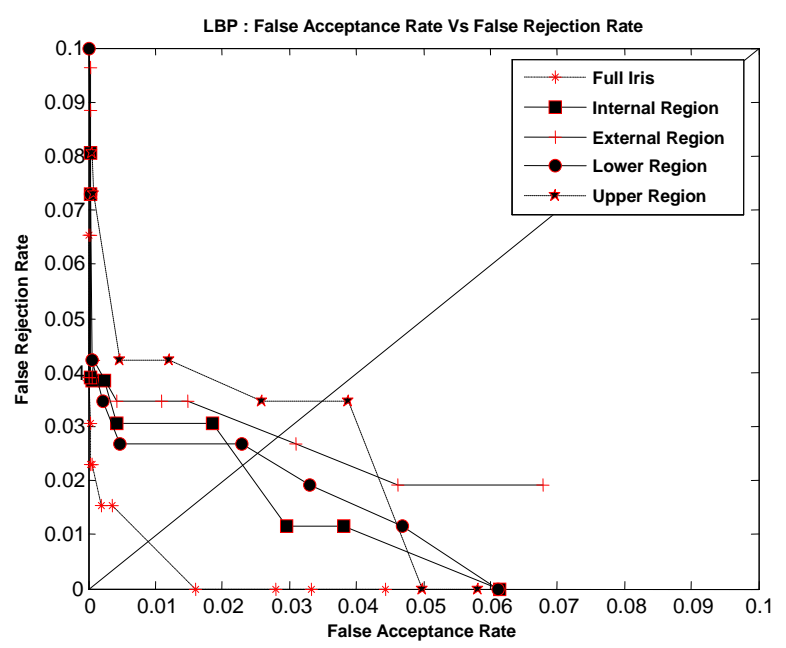

Fig 5: Local Binary Pattern: ROC Curve

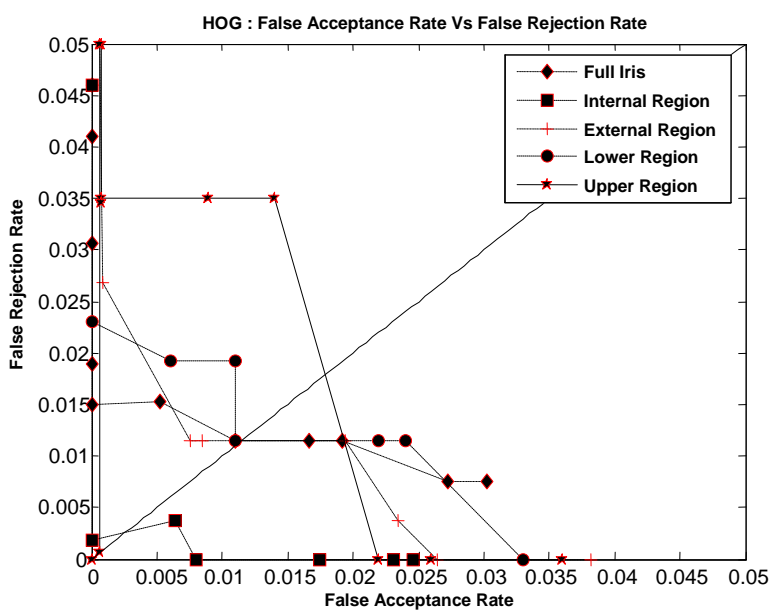

Fig 6: Histogram Oriented of Gradient: ROC Curve 


\section{CONCLUSION}

This paper has investigated the comparative performance from different approaches on feature extraction for the iris identification: entire iris template, half template and template of half of the iris portion. The iris features are concentrated along regions near the pupil. Half Iris feature extraction methods can find the iris characteristic points in a short time, the recognition rate is high, and the recognition speed is guaranteed because of reduction in its dimensionality and well suited for natural open eyes. This experiment is applicable to do experiments on a larger iris database in various environments for iris recognition system.

\section{REFERENCES}

[1] Daugman J, “Complete Discrete 2-D Gabor Transforms by Neural Networks for Image Analysis and Compression", IEEE Transactions on Acoustics, Speech, and Signal Processing, Vol. 36, no. 7, July 1988, pp. 1169-1179.

[2] Daugman, J. "How Iris Recognition Works", available at http://www.ncits.org/tc_home/m1htm/docs $/$ m1020 044.pdf.

[3] Daugman.J, "Biometric Personal Identification System Based on Iris Analysis" U.S.Patent No. 5,291,560 issued March 1, 1994.

[4] J.G.Daugman, "High Confidence visual Recognition of Persons by a Test of Statistical Independence", IEEE transactions on Pattern Analysis and Machine Intelligence, Vol. 15, No. 11, pp. 1148-1161, 1993.

[5] K.Levi and Y.Weiss,"Learning object detection from a small number of examples: the importance of good features", IEEE CS Conference on Computer Vision and Pattern Recognition, pp. 53-60, 2004.

[6] N.Dala and B.Tiggs, "Histograms of oriented gradients for human detection", IEEE CS Conference on Computer Vision and Pattern Recognition, pp.886893, 2005.

[7] B. Wu and R. Nevatia, "Detection of multiple, partially occluded humans in a single image by Bayesian combination of edgelet part detectors", IEEE CS Conference on computer Vision and Pattern Recognition, pp. 90-97, 2005.

[8] L. Masek, "Recognition of Human Iris Patterns for Biometric Identification" M.Thesis, The University of Western Australia, 2003, www.csse.uwa.edu.au/ pk/studentprojects/libor/Libor MasekThesis.pdf, Mar. 26,2005.

[9] T.Ojala, M.Pietikainen and D.Harwood. A comparative study of texture measures with classification based on feature distributions. Pattern Recognition, January 1996.

[10] J. Huang, Y. Wang, T. Tan, and J. Cui “A New Iris Segmentation Method for Recognition", Proceedings of the 17th International Conference on Pattern Recognition, 2004.
[11] MMU Iris Image Database: Multimedia University, http://pesonna.mmu.edu.my/ ccteo/

[12] J. Daugman, "Face and gesture recognition: Overview", IEEE Transactions on Pattern Analysis and Machine Intelligence, Vol 19, No.7, pp, 675-676, 1997.

[13] H.Wang and S.F.Chang, "A Highly Efficient System for Automatic Face Region detection in MPEG video", IEEE Transactions on Circuits and Systems for Video Technology, Vol. 7, No. 4, pp.615-628, 1997.

[14] A.Poursaberi and B.N.Araabi,"A Half-Eye Wavelet Based Method for Iris Recognition", IEEE Proceedings of , $5^{\text {th }}$ International Conference on Intelligent Systems Design and Applications, 2005 (ISDA'05).

[15] L. Ma, T. Tan, Y.Wang and D.Zhang,"Efficient Iris Recognition by Characterizing Key Local Variations",IEEE Transactions on Image Processing, Vol. 13, No.6, 2004, pp, 739-750.

[16] T.Tan, L.Ma, Y.Wang and D.Zhang," Personal Identification based on Iris Texture Analysis", IEEE Transactions on Pattern Analysis and Machine Intelligence, Vol.25, No. 12, 2003, 1519-1533.

[17] Maheswari, P.Anbalagan and T.Priya, "Efficient Iris Recognition through Improvement in Iris Segmentation Algorithm", ICGST-GVIP Journal, ISSN: 1687-398X, Vol 8, Issue 2, pp. 29-35, 2008.

[18] L.V.Birgale and M.Kokare, "Iris Recognition Using Discrete Wavelet Transform", IEEE Proceedings of the International Conference on Digital Image Processing (ICDIP'09), 2009.

[19] G.Savithiri and A.Murugan,'Performance Analysis of Iris Recognition Algorithms", Proceedings of the International Conference on Millennium Development Goals (MDGICT) pp. 294-299, Dec. 2009.

[20] G.Savithiri and A.Murugan "Iris Recognition Technique using Gaussian Pyramid", Proceedings of the International Conference on Recent Trends in Business Administration and Information Processing, (BAIP), CCIS 70, pp-325-331, Springer-Verleg Berlin Heidelberg, March 2010.

[21] G.Savithiri and A.Murugan, "Iris Recognition Technique using Gaussian Pyramid Compression and Modified Distance Measures", Journal of Computational Intelligence in Bioinformatics, Vol 3, No. 1, July 2010, pp 101-110.

[22] G.Savithiri and A. Murugan. Feature Extraction on Half Iris for Personal Identification, Proc. IEEE International Conference on Signal and Image Processing (ICSIP), pp 197-200, Dec 2010. 